Revue de l'Institut des langues et cultures

d'Europe, Amérique, Afrique, Asie et Australie

$45 \mid 2022$

Femme(s) et sorcellerie en Espagne et en Italie à l'époque moderne

\title{
Pierre de Lancre, Pedro de Valencia : deux visions du procès de Logroño
}

Pierre de Lancre, Pedro de Valencia: Two Visions of the Trial of Logroño

Renaud Cazalbou

\section{OpenEdition}

Journals

Édition électronique

URL : https://journals.openedition.org/ilcea/14590

DOI : $10.4000 /$ ilcea. 14590

ISSN : 2101-0609

Éditeur

UGA Éditions/Université Grenoble Alpes

Édition imprimée

ISBN : 978-2-37747-330-4

ISSN : 1639-6073

Référence électronique

Renaud Cazalbou, «Pierre de Lancre, Pedro de Valencia : deux visions du procès de Logroño », ILCEA

[En ligne], 45 | 2022, mis en ligne le 31 janvier 2022, consulté le 31 janvier 2022. URL : http://

journals.openedition.org/ilcea/14590; DOI : https://doi.org/10.4000/ilcea.14590

Ce document a été généré automatiquement le 31 janvier 2022.

(c) ILCEA 


\title{
Pierre de Lancre, Pedro de Valencia : deux visions du procès de Logroño
}

Pierre de Lancre, Pedro de Valencia: Two Visions of the Trial of Logroño

\author{
Renaud Cazalbou
}

«Apres tout: c'est mettre ses coniectures à bien haut pris, que d'en faire cuire un homme tout vif ", écrivait Montaigne, illustrant ainsi ce qui semble être au cœur de la question de la sorcellerie en Europe entre les $\mathrm{Xv}^{\mathrm{e}}$ et $\mathrm{XVII}^{\mathrm{e}}$ siècles. Qui croire ? Ceux qui prétendent que ces récits ne sont que des fables, peut-être dues à l'invention du démon, qui ne ferait, dans le fond, que ce qu'il a accoutumé de faire : semer le trouble, jouer des illusions pour tromper les hommes, en un mot mentir, conformément à l'un des sens étymologiques porté par son nom, « le calomniateur ${ }^{1}$ »; ou bien ceux qui tiennent pour véritables les récits de ces femmes qui se disent sorcières et déclarent être allées, de nuit, à la rencontre du démon dans des assemblées où l'on pratiquait le blasphème, la luxure et le meurtre? Ces deux avis sont représentés par les deux personnes qui figurent dans ce titre. D'un côté, Pierre de Lancre, conseiller au parlement de Bordeaux, envoyé par Henri IV en pays de Labourd " pour la recherche du crime de Sorcelerie ", qui revendique le parti le plus radical : "Qu'il fallait faire mourir les sorcières pour avoir été simplement au sabbat, quand bien même elles ne seraient prévenues d'aucun maléfice. » (Lancre, 1612 : 566) De l'autre, Pedro de Valencia qui écrit en 1611, comme il le dit lui-même, un "intento del examen y censura de aquellos cuentos: y no hay cosa que tanto lo desacredite como las monstruosidades increibles $y$ incompatibles que contienen " (1611a: fol. 1v). Les deux auteurs, pourtant, traitent de la même matière, racontent le même événement et s'appuient sur le même document : le récit du procès de Logroño des 6 et 7 novembre 1610 où sont jugés, notamment, des sorciers et sorcières du Pays basque, plus particulièrement de la région de Zugarramurdi. Il s'agit de la Relación de las personas que salieron al Auto de Fe que los señores don Alonso Becerra Holguin, del hábito de Alcántara, licenciado Juan Valle Alvarado, y licenciado Alonso de Salazar y Frias, inquisidores apostólicos del reino de Navarra y su distrito, celebraron en la ciudad de 
Logroño en 7 y 8 dias del mes de noviembre de 1610 años, $y$ de las cosas y delitos por que fueron castigadas, publiée à Logrogne en 1611 (l'approbation est datée du 6 janvier, la licence accordée à l'éditeur Juan de Mongastón, du 7 janvier). La rapidité de l'édition de cet opuscule qui, certes, n'arrive pas à trente pages, est la preuve de l'intérêt que portent les lecteurs à ces histoires de sabbat, de démons et de sorcières. Deux visions d'un même événement, donc, mais aussi deux positions quant à l'attitude à avoir face à ce que l'époque appelle « l'hérésie des sorcières ».

2 Il est d'évidence que Pierre de Lancre accorde le plus grand crédit aux " témoignages » et "aveux » des sorcières et ce qu'il pense de la Relación peut être résumé par ses propres mots, au moment où il entre dans le vif de son récit : "Or affin qu'on recognoisse les grandes meschancetez qui se commettent par les sorciers ie mettrai icy ce qui se passa en cet acte de la foy. " (Lancre, 1612:387) On peut s'arrêter un instant sur la matérialité de la transcription : les cinq premières pages du discours III du livre $\mathrm{V}$ du Tableau de l'inconstance des mauvais anges et démons, où il est amplement traicté des Sorciers et de la Sorcellerie sont une traduction littérale, ou peu s'en faut, de la Relación, laquelle est annoncée dès le titre général ${ }^{2}$ de l'ouvrage: Avec un discours contenant la procédure faicte par les Inquisiteurs d'Espagne \& de Navarre, à 53. Magiciens, Apostats, Iuifs \& Sorciers, en la ville de Logrogne en Castille, le 9. (sic) Nov. 1610. On ne signalera que quelques divergences qui sont sans doute plus des erreurs que de réelles modifications : "con Sambenito y Coroças de Relaxados" dans la Relación traduit une première fois par "qu'ils avaient été relaxez d'autrefois de l'inquisition" et une deuxième fois par le simple " relaxés » qui semble être un contre sens assez surprenant. En effet, de Lancre confond les verbes espagnol "relaxar » et français "relaxer». La différence est de taille, car si "relaxer» ou "rélaxer» a le sens que nous lui connaissons aujourd'hui dans le vocabulaire de la justice, le verbe espagnol signifie dans l'acception du texte "abandonner au bras séculier » et équivaut donc à une condamnation à mort. De même, l'expression «cristianos nuevos de judíos " a-t-elle été rendue par l'étonnante "six chrestiens nouveaux des Indes». Enfin, la proposition "Otro, por haber sido luterano, creyendo y teniendo proposiciones de la secta de Lutero" devient, chez de Lancre « un autre, pour avoir été empoisonné de l'hérésie de Calvin, et soutenu quelques propositions de Luther... ». Ces quelques distorsions mises à part, la traduction française, des pages 382 à 387 de l'édition de 1612, est le calque de l'original espagnol.

3 Il s'agit donc aux yeux mêmes de Pierre de Lancre d'une partie notable, voire capitale de son livre. En faisant figurer dans le titre de l'ouvrage la référence à ce procès et en donnant à ce récit une place centrale dans son traité, il justifie et légitime la chasse aux sorcières qu'il mène en pays de Labourd. Le procès de Logrogne est donc pour lui l'illustration des crimes (véritables) commis par les suppôts du diable. De là l'importance accordée au témoignage qui n'est pas, sans doute, uniquement de la déformation professionnelle. Il est, en tant que conseiller au parlement de Bordeaux, mandaté par Henri IV pour "purger le pays de tous les sorciers et sorcières sous l'emprise des démons » en pays de Labourd. Or, selon les usages judiciaires de l'époque, le témoignage et l'aveu sont des pièces capitales. En matière de démonologie, c'est bien souvent la seule preuve, au sens de la pistis aritotélicienne, c'est-à-dire ce qui est à même d'entraîner la conviction. En effet, dans la doxa des démonologies, il est courant que le démon cache les traces de ses œuvres comme en témoigne cette anecdote de la Relación, évidemment citée par le même de Lancre, au sujet de María de 
Yurreteguia. Initiée fort jeune à la sorcellerie, elle avait cependant voulu s'en éloigner et s'était attiré la colère de ses anciennes compagnes :

Mais comme elles la virent ferme \& constante embrassant la Croix, elles se retirerent, avec une telle violence pourtant, qu'elles luy firent esbranler toute sa maison, luy gastèrent tout son jardin: \& pour montrer plus leur rage, elles prindrent un moulin avec ses meules qui estoit là voisin, le portèrent et le mirent au-dessus d'une montagne, auprés duquel ayant lõguement dancé, ils le remirent en son lieu, mais tout en desordre \& en pieces. (De Lancre, $1612: 388$ )

4 De ce phénomène prodigieux, le déplacement d'un moulin en haut d'une montagne, et quand bien même ce ne serait qu'un « cerro » comme le déclare la Relación, il ne reste de trace que quelques dégâts, certes importants, mais sans commune mesure avec l'événement relaté : «[...] volvieron todo el molino entero como lo llevaron, y los demonios lo pusieron y concertaron como estaba, dejando roto el rodezno y el husillo en el agua, y la piedra molar á un lado. » (1611 : fol. 5-6) Dans la Suma de las relaciones de Logroño cerca de Brujos (ms. $n^{\circ} 7579^{3}$ de la BN de Madrid, fol. 1-19v), Pedro de Valencia cite la même anecdote : "[...] le arrancaron todo un molino de su lugar, jurándose á hacer esto los brujos, y muchos diablos y lo pusieron encima de un cerro; $y$ que lo volvieron a poner en su lugar la misma noche, dejando roto el rodezno, el husillo en el agua, y la piedra molar á un lado ", qu'il assortit d'une note «Mal hayan los diablos porque no lo dejaron sobre el monte como Sanson las puertas de la ciudad, para que creyeran todas estas valentías. Lo del rodezno, husillo y piedra, quatro brujos lo pudieron hacer » (1611a : fol. 7r). Si le Discurso se veut une œuvre policée, aux arguments scientifiques, la Suma, plus ramassée, plus travaillée aussi, joue de notes ironiques ajoutées à un résumé de la Relación en apparence objectif. Ici, le simple fait de signaler que les démons, dans le fond, ne sont pas très rusés - on n'ose dire pas très malins suffit à désamorcer l'anecdote et à lui ôter toute vraisemblance.

5 Les deux textes n'ont sans doute pas la même visée et ils diffèrent donc par le ton qui les définit. Le Discurso, adressé à Bernardo de Sandoval y Rojas, archevêque de Tolède et inquisiteur général obéit, certes, au devoir de solennité requis par le prestige de son destinataire mais, en outre, il revêt un caractère officiel car Pedro de Valencia est mandaté par l'archevêque pour formuler un avis sur le contenu de la Relación : «[...] y aviendo V. S. Ill ${ }^{\text {ma }}$ servídose, no sole darme licencia sino mandato, obedeciendo, y sometiendo mi parecer en todo a la censura de nuestra universal madre y maestra la santa Iglesia Cathólica Romana, V. S. Ill ${ }^{\text {ma }}$ digo. » (1611a : fol. 3r). Au contraire, la Suma est un texte qui n'a pas de destinataire précisément identifié et, par là même, il échappe à une tonalité imposée. Bien que la date n'en soit pas connue avec certitude, il est cependant intéressant de signaler qu'il est postérieur au Discurso du 20 avril 1611. En effet, Pedro de Valencia déclare à la première page de la Suma: "Esto fué causa de que un discurso que para un examen escribí $y$ ofrecí al Ill ${ }^{m o}$ Cardenal Arçobispo de Toledo mi señor, fuese menos cumplido.» (1611a : fol. 1r) La Suma est donc considérée par l'auteur lui-même comme un travail plus abouti, ce qui montre que l'humour, la raillerie, voire le sarcasme dont il fait usage à l'égard du contenu de la Relación sont autant d'outils destinés à déconstruire et à disqualifier le discours sur les sorcières. L'analyse discours verrait dans ces deux textes, le Discurso et la Suma, l'opposition de deux éthos: dans le premier, on voit se contruire un éthos de gravité voire d'orthodoxie, lié à la mission qui est confiée à l'auteur, alors que dans le second, c'est un éthos de combat qui se met en place.

6 Si l'on se penche sur le contenu des textes et sur les propos rapportés, on verra que le traitement apporté par de Lancre est au rebours de ce qui vient d'être dit: lorsqu'il évoque le fait que le moulin est revenu à sa place, il déclare qu'ils «le remirent en son 
lieu, mais tout en desordre \& en pieces ». Chacun comprendra que l'auteur, ici déplore un événement qui, pour lui, s'est réellement passé et qui est la marque de la grande puissance des sorciers. Cette impression a une cause qui est la structure argumentative de la proposition, et tout particulièrement, l'utilisation du connecteur mais. Ce dernier fonctionne toujours de la même façon: l'argument qui suit mais, conformément à l'étymologie de cette conjonction (magis), a une force supérieure à celui qui précède. Ainsi que l'a analysé 0 . Ducrot, cela revient à minimiser la portée de ce qui précède mais : «[...] le locuteur déclare négliger le premier [énoncé] dans l'argumentation qu'il est en train de construire, et s'appuyer seulement sur le second - la force argumentative supérieure accordée à celui-ci n'étant qu'une justification de cette décision. » (1983: 9) Or ici, Pierre de Lancre joue de cette propriété. En déclarant que le moulin est revenu à sa place MAIS «tout en desordre \& en pieces », il limite le fait que rien ne vienne prouver que ce moulin a effectivement été transporté. Un même « incident », deux interprétations différentes. L'usage de la langue est ici révélateur d'un état d'esprit, celui d'un homme prêt à croire dur comme fer ce qui sera déclaré, avoué, confessé y compris les choses les plus fantastiques. En veut-on un autre exemple?

7 Au sabbat, les sorciers, si l'on en croit la Relación, disposent d'une plante susceptible d'amollir les os des cadavres déterrés qui ont servi à des festins macabres :

La primera vez que después vuelven al aquelarre echan á cocer los huesos del difunto que comieron antes, $y$ con ellos las hojas, ramas y raices de una yerba que en vascuence llaman Belarrona, que tiene virtud de ablandar los huesos y los pone como si fueran nabos cocidos; [...]. (1611:29)

Dans le texte de Pierre de Lancre, la chose est transcrite de la façon suivante : «\& pour les os, ils les mettent dans des pots iusques à la nuict ensuivãt, en laquelle ils les fõt cuire avec une certaine herbe que le Diable leur monstre, laquelle les rend aussi tendres que des naveaux.» $(1612: 395)$ On serait tenté de dire qu'il y a là une traduction relativement fidèle du texte espagnol; en tout cas, l'essentiel de l'information est conservé jusqu'à la comparaison avec les navets. La transcription de Pedro de Valencia semble être du même acabit et reprendre le texte de la Relación: "Añaden mas que los huesos desta carne se guardan para el siguiente aquelarre, y que los cuecen con cierta yerba llamada en vascuenço belarrona, que tiene virtud de ablandarlos como nabos.» (1611a: fol. 17r) À la réserve près que le mot « nabos » est assorti d'un appel $\left(n^{\circ} 42\right)$ qui renvoie à la note marginale suivante : "Si esta virtud es natural, de grande importancia sería para la republica si se aprovechasen los huesos. " Là encore, la saillie disqualifie le texte. En feignant de se placer sur le terrain de l'utilité publique, en faisant usage, pour tout dire de bon sens, Pedro de Valencia oblige le lecteur à revenir à une appréciation juste des choses. En d'autres termes, il oblige ce même lecteur à quitter le champ du fantastique pour revenir au prosaïsme des préoccupations quotidiennes. On le voit, cette démarche, simple, concrète n'a rien à voir avec une réfutation théologique mais, tout au contraire, confronte le discours à l'évidence de la réalité.

9 Ces aspects à la fois sordides et pittoresques du sabbat occupent une large place dans la Relación, en voici un autre exemple: les sorciers prétendent que les reliefs de ce repas sont utilisés pour fabriquer des poisons. Soit le texte de la Relación :

[...] y una parte de ellos comen, y otra el demonio y brujos mas ancianos la machan en unos morteros, $y$ los esprimen con unos paños delgados, $y$ sacan de los dichos huesos una agua clara y amarilla que el demonio recoge en una redoma; [...] Y es tan grande la ponzoña $y$ 
fuerza de aquella mala agua, que tocando con ella cualquiera persona en cualquier parte de su cuerpo, con mucha brevedad, muere sin que haya remedio humano para ello. (1611:15) laquelle ils pressent entre deux pierres, \& en tirent une liqueur mortelle. » Certes, l'eau jaunâtre est-elle devenue une "paste", mais on ne peut parler de divergence fondamentale. D'ailleurs le texte de la Relación fait aussi allusion à des poudres et poisons réalisés à partir des « cisco que queda de los huesos y los sesos de los difuntos ». On considérera donc que de Lancre a choisi de résumer, de concentrer le propos sans toutefois en modifier ni atténuer le sens. Pedro de Valencia semble abonder dans ce sens et décrit la chose de la façon suivante, au plus près semble-t-il, du texte source :

Que parte dellos comen, parte machacan el demonio y los brujos en un mortero $y$ exprimiéndolos entre paños, recoge y guarda el demonio en una redoma el agua que vale, que es clara y amarilla; y el cisco destos huesos y los sesos que sobran los recogen los demonios menores y hacen dellos unos polvos muy ponzoñosos. Que ${ }^{43}$ el agua amarilla es de mucha estima, porque es de presentísimo y eficacísimo veneno mortal. (1611a : fol. 18r-v)

11 À nouveau, un appel de note $\left(\mathrm{n}^{\circ} 43\right)$ qui renvoie au texte suivant: "Maravilla es que de huesos o nabos tan blandos de comer salga agua tan venenosa. " C'est encore l'argument de bon sens qui est convoqué pour provoquer un sourire ironique. Cet aliment, dont on ne sait plus s'il s'agit d'os ou de navets, ne saurait être un poison puisqu'il a aussi servi d'aliment, lors du festin, sans incommoder aucun sorcier et pourtant le jus qui en sort peut tuer par le seul contact. Là où le démonologue voit la marque du diable, la personne sensée voit une contradiction majeure, irréductible. Il en va de même, lorsque dans la Relación, il est question d'un chaudron de cuir : «llevando el dicho Miguel de Goyburu la caldera del demonio, que es de cuero, donde se ha recogido gran parte de los dichos polvos para el dicho efecto" (1611:29). Pedro de Valencia ajoute le commentaire ironique : «Notable desatino. Otra vez nos dirán si la sarten es de paño o de lienço.» (1611a : note 39 , fol. 14r) évident que Pedro de Valencia a pris le parti de montrer combien le texte de la Relación et, au-delà, le discours de la démonologie sur les sorcières, est peu vraisemblable, fantastique, irrationnel. Bien qu'il ait averti le lecteur, à l'ouverture de la Suma, qu'il s'agit de «cuentos", il lui importe de le prouver. Pour ce faire, la première des armes est le recours au bon sens, l'appel à la raison et à la lucidité du lecteur par le biais de l'humour, ce qui constitue une irruption de la subjectivité dans un texte qui se veut impartial, objectif ou pour le moins qui se présente comme tel. Aussi, dans la Suma, au moment où il évoque le banquet diabolique du sabbat, place-t-il cette réflexion significative :

Esto es ageno de toda verisimilitud, y aun creerlo es cosa inhumana, porque excede en abominacion y crueldad horrenda todo lo que los poetas se alargaron á fingir de los ciclopes y lestrigones, y quanto se cuenta de los brasiles y cáribes antropófagos. (1611a : fol. 17r)

On voit ici se développer le deuxième procédé de réfutation utilisé : il s'agit de la connaissance et de l'érudition. Le renvoi à la dimension mythologique, d'une part, et à la situation du Nouveau Monde, d'autre part, constitue un argument d'autorité supposé à même de contrer la force de persuasion du discours ambiant. Toutefois, on aurait tort de croire que ces deux arguments sont à mettre sur le même plan : dans les deux cas, il s'agit, certes, de choses racontées, de discours rapportés, mais la distinction entre ce qui est dit du monde réel et ce qui est une invention littéraire (" fingir ») des poètes est maintenue et affirmée. De plus, on remarquera que le discours de Pedro de Valencia est 
clairement polyphonique: si les notes constituent un contrepoint humoristique qui vise à discréditer les événements relatés, le corps du texte est, lui, le plus souvent réservé à une argumentation réfutative plus orthodoxe. On verra qu'il n'en va pas toujours de même.

14 À l'inverse, le discours des démonologues présente une force insoupçonnée: les confessions de sorcières, comme les traités théoriques, fourmillent de contradictions, de fautes logiques, qu'il s'agisse de récits fantasmagoriques ou d'impossibilités physiques. Toutefois, ce discours a survécu et a gagné en adeptes en persuadant les lecteurs de sa véracité. Précisons qu'il serait trop simple de prendre les tenants du discours démonologique comme peut l'être de Lancre, soit pour des sots, soit pour des sadiques (ce qu'ils peuvent être bien évidemment) uniquement préoccupés d'assouvir leurs pulsions de cruauté. Comment, dès lors, peut-on interpréter l'opposition radicale entre la position de Pedro de Valencia et celle de Pierre de Lancre? Dans le Discurso sobre los cuentos de las brujas, le premier affirme :

Muy fea ignorancia descubre el hombre letrado que niega esto; ansi que no se tome por señal de catolico la facilidad en admitir estos cuentos, que antes los manicheos persas y moros son los que atribuyen mucho poder al demonio: y el que es fanfarron desea que se la atribuya mas y mas: [...]. (1611b : fol. 56r)

Les crapauds parlants du sabbat, la possibilité donnée aux sorcières de franchir les portes closes, les poudres qui font perdre les cultures, etc., tout cela est traité dans la Suma de la même façon, c'est-à-dire sur le ton du mépris :

Este es un cuento insufrible por su irreverencia y obscenidad, $y$ del todo es fingido o descubre que todo es bellaquería, y imitación hecha por hombres. (1611a : fol. 10v) Mais ce que l'on ne peut nier, c'est que le discours des démonologues est avant tout une formidable construction théorique et formelle qui ne demande qu'une chose : que l'on en adopte le présupposé fondamental selon lequel le démon doit être considéré, à l'instar du poumon dans le Malade imaginaire, comme cause de tout et de son contraire, car là est sa marque. Cela, Pedro de Valencia l'a très bien senti, qui montre que les gens enclins à croire ces fantaisies iront toujours vers l'explication prodigieuse : si un enfant meurt de mort naturelle, le vulgaire ira chercher et, plus grave, trouver les preuves de causes surnaturelles: "[...] y en los que mueren ansi suelen hallarse manchas $y$ como cardenales y ronchas por todo el cuerpo o por algunas partes dél, como saben los médicos; y estas señales dirá el vulgo que son pellizcos de la bruja. » (1611b : fol. 70v)

Cette gradation en effet ne concerne pas seulement l'espace intra-discursif, mais aussi tout le référentiel; si l'on va vers plus de précision et d'horreur dans la description, parallèlement, n'importe quelle réalité peut être prise comme un signe d'appartenance au diable au point même que l'absence de signe a fini par devenir suspecte : on sait que, dans la littérature démonologique, la marque de Satan est une preuve irréfutable de sorcellerie, mais que dire alors de ces auteurs qui prétendent que lorsqu'une sorcière n'a pas de marque c'est que le démon l'a ôtée ; cela revient à dire que l'indice comme l'absence d'indice sont tout aussi probants. Dans la Relación, il est dit que le diable appose deux marques sur le corps des sorciers : «[...] y después en la niñeta de los ojos con una cosa caliente, como si fuese de oro, le marca (sin dolor) un sapillo, que sirve de señal con que se conocen los brujos unos á otros. " Ce qui chez de Lancre devient : «\& tout aussi tost prenant une epingle d'or faux, il les marque le plus souvent dans le blãc de l'œil gauche, \& leur imprime une marque qui semble un petit crapaud.» Or, cette marque qui serait une preuve objective irréfutable, personne ne peut la voir, ce qui n'est d'ailleurs 
explicitement déclaré que chez Pedro de Valencia qui relève dans la note 8 : «Parece que quiere decir que este sapillo no es visible para los que no son brujos. » En d'autres termes, et on appréciera l'oxymore, il est traité d'une marque invisible. Le discours sur les sorciers fait ici la preuve de son appartenance à la grande famille des discours totalitaires de réprobation et d'accusation. Dans une situation de ce type, comme le souligne Kerbrat-Orecchioni, au sujet du procès de la bande des Quatre en Chine, «le degré de péjoration joue un rôle déterminant, mais le contenu dénotatif n'importe guère : on assiste alors à une sorte de vidage sémantique de l'expression au profit de la seule connotation axiologique» $(1980: 80)$. Le mécanisme est simple et remarquablement efficace : tout vocable, tout propos, du fait même qu'il est intégré dans le discours, contribue à renforcer l'accusation. De fait, les mots, vidés de leur contenu sémantique, sont réduits au statut de simples marqueurs. On pardonnera la comparaison, mais le procédé est similaire à celui qui est utilisé dans les insultes du capitaine Haddock: " anacoluthe", "catachrèse " ne deviennent insultant que dans ce seul contexte. De même, dans le discours démonologique, accuser une femme ou un homme de sorcellerie, c'est la ou le faire entrer dans ce schéma discursif où tous les arguments sont subordonnés à cette prémisse. Peu importera donc la vérité des accusations portées, toutes seront sollicitées en vue de la confirmation de cette affirmation première. C'est là une façon d'abolir toute contradiction. D'une certaine manière, on peut considérer que le discours démonologique est moins dénotatif que connotatif.

Semblablement, les sorcières de Logrogne disaient que lorsqu'elles déterraient les morts, elles remettaient tout en place au point que l'on ne pouvait voir trace de leur passage : «Ansi pueden decir que la noche passada trasladaron toda esta villa, y quanto en ella está al puerto de Guadarrama, y la bolvieron a assentar en su lugar sinnadie lo sintiesse.» (Valencia, 1611b: fol. 72r) Le bon sens ne peut trouver qu'une solution à cette confusion : «Si uno depusiesse ante el juez contra Pedro, ó confessasse contra sí que avia ido en una hora a Mexico y muerto allí a puñaladas à un su enemigo, mas razon era atar y curar à este hombre, que darle crédito, ni prenderlo. " (Valencia, 1611b: fol. 70r) «Dar crédito ", voilà bien encore et toujours le maître mot. Dès lors, le discours de Pedro de Valencia scinde en trois grands groupes les domaines cognitifs de l'homme avisé : il y a l'histoire sacrée à laquelle on doit la foi ( se debe toda $f e »)$, l'histoire humaine à laquelle on peut parfois accorder crédit ("dar crédito ») et les déclarations de ces femmes qui ne sont que billevesées («cuentos»). Il propose la distinction des plans épistémiques afin de juger chaque corpus à l'aune qui lui correspond. Or, la prose démonologique à des fins évidentes, avait tout regroupé sous le même article de foi qu'il s'agisse d'Homère, du prophète Daniel ou des déclarations d'accusés et accusées. On peut, à la lumière des présupposés scientifiques actuels, se gausser de cette confusion, pourtant ce serait manquer l'une des caractéristiques majeures du discours: que, parmi les démonologues, il y en ait eu pour lire de façon ingénue Ovide ou Apulée, croyant que leurs écrits étaient véridiques, cela ne fait aucun doute, mais ce n'est pas le cas de tous ; en revanche, le commun dénominateur est l'unification de l'univers de croyance et de savoir. Par là, le discours démonologique acquiert la portée universelle dont il manque par nature. En effet, ses sources ne sont que les élucubrations de quelques femmes bien souvent incultes, car ce sont elles qui s'accusent de ces crimes. Un cadre théorique et historique fait cruellement défaut; le seul moyen de donner une assise à la démonologie consiste donc à lui attribuer des autorités capables de lui assurer une légitimité. L'histoire sacrée, objet de foi, en est une et l'Antiquité, qui est le fondement de toute la culture, une autre; les aveux des sorcières et sorciers confirment ce corpus. 
Mais on notera le renversement; ce que dit la prétendue sorcière est tout à la fois principe et confirmation. C'est dans ce courant que se place Pierre de Lancre.

Tant y a que le Diable est le maistre des souplesses, \& quelque diversité qu'il employe pour eluder le monde, il tend neantmoins tousiours a un mesme but, qui est de detruire entierement le genre humain : cest pourquoy il se sert de tãt de visages, de tãt d'illusions, de tant de fourbes, \& ne les estale guiere jamais qu'en tenebres, comme instrument tres-propre de surprise. $(1612: 398)$

De fait, puisque le diable est un maitre d'illusion, il pourra, dans cette perspective, jouer des contraires pour arriver à ses fins, ce qui a pour conséquence de rendre crédible toute affirmation et son contraire du moment que l'on y voit la patte du démon. «Le poumon, ou plutôt le démon vous dis-je. » De ce point de vue, le discours démonologique sur les sorcières présente un aspect fortement performatif, car la seule évocation du diable suffit à le rendre crédible et légitime, à lui donner sa justification. Sous cette autorité, car le diable en est une dans ces discours, tout peut être dit et tout finit par faire sens. Or, Pedro de Valencia attaque le problème à la racine. Si l'on a pu dire qu'il construit une véritable analyse du discours, c'est qu'il se préoccupe plus de cohérence discursive que de trouver des arguments de réfutation: la thématique, les conditions de production du discours, l'analyse de séquences révélatrices de la logique qui sous-tend le discours, tout cela est étudié, disséqué, analysé. Mais un aspect fondamental de ces textes - et plus particulièrement du Discurso - en est le fil directeur : l'interrogation sur les domaines de croyance et de savoir. La question est primordiale, car elle fait appel à la portée épistémique du discours : que croire ? et que sait-on? sont les questions qui structurent tout le propos. À l'issue de la démarche, il demeure une vision très stratifiée qui sépare l'acte de foi, l'opinion et le savoir. L'exception espagnole est sans doute à découvrir dans cette distinction. Comme le dit Pedro de Valencia lui-même, l'Espagne est un pays d'une très grande orthodoxie catholique (il parle de "Reynos tan puros de heregía»). On peut aisément concevoir qu'une foi solide alliée à une grande discipline et un rite sûr de lui aient été un bon moyen de se prémunir contre l'hérésie des sorcières. Or, les plus grands foyers de sorcellerie correspondent aux zones et régions qui ont été travaillées par des velléités de scissions, de schismes... De là, naît un discours de la citadelle assiégée, celui de la démonologie.

$\mathrm{Au}$ contraire de lui, de Lancre ne procède pas à cette mise à distance. Il accepte sans sourciller les dires des accusés sans même se rendre compte que c'est par sa plume que se fixent les stéréotypes. La justification qu'il donne de ce III discours est, à cet égard, tout à fait révélatrice :

Il m'a semblé estre fort à propos pour contenter la curiosité du lecteur, de coucher icy non seulement les traicts nouveaux, estranges \& espouvãtables, que le Diable faict faire aux sorciers au sabbat \& ailleurs, en Espaigne \& Navarre, mais encore la forme, qu'ils ont tenu à leur faire le procez. Ce que i'ay tiré de la Relation d'Espagne, \& du propre imprimé qui a esté faict à Logrogne qui m'est tombé en main. (1612: 382)

21 Si l'on a parlé de « performativité » du discours sur les sorciers, c'est aussi parce qu'il a cette capacité à s'auto-produire et s'auto-reproduire par le biais de l'intertextualité. À l'un des arguments avancés par les démonologues, tel Jean Bodin, selon qui la similitude des aveux de toutes les sorcières d'Europe était la preuve de la réalité du sabbat et de l'existence des sorcières, Pedro de Valencia oppose l'argument inverse en 
se fondant sur l'existence non pas de faits récurrents impossibles à vérifier mais d'un discours stéréotypé largement répandu :

Y aunque ayuda a persuadir esto la conformidad de los dichos de todos en tales disparates, que da á entender que es cosa aprendida, y que pasa por tradicion de mano en mano, de provincias en provincias y de siglos en siglos, que de otra manera no fuera cundiendo en los vecinos el contagio, sino que el demonio lo enseñara de salto en regiones apartadas, y tambien inventara cada dia nuevas ceremonias en su adoracion y servicio. (1611b: fol. 70r)

Bien avant Ginzburg (1992), la possibilité d'un substrat folklorique est ici évoquée, mais plus encore la vision du cheminement d'un discours, d'une diffusion de bouche à oreille dans un contexte géographique relativement limité qui peut s'apparenter au mécanisme de la rumeur. Il s'agit encore et à nouveau, chez celui qui est souvent qualifié d'humaniste, de chercher et de trouver l'humain, le naturel. Car ces histoires de sorcières, de sabbats, d'assassinats rituels ne sont que cela, du discours qui s'étend au gré de l'histoire et des mouvements de populations. D'ailleurs Pedro de Valencia soulève un point capital: jamais aucun auteur de traité, aucun inquisiteur n'a d'expérience des faits qu'ils doivent juger, « todos se refieren a oídas ». Quand, par hasard, un auteur, comme le Père del Río, se réfère à une anecdote personnelle ${ }^{4}$, il s'agit d'un emprunt au motif folklorique de l'animal blessé qui s'avère une personne, un élément discursif, donc. Le jugement ne se fait pas attendre : «Es de ver y admirarse de un hombre que cuenta esto tan en juicio. [...] Es nunca acabar, et cum iudicio insanire, querer reducir á raçon y verissimilitud lo que se cuenta deste género. » (Valencia, 1611b : fol. 74r)

Mais il y a plus, si l'on y regarde de près, on peut, parfois, voir affleurer les mécanismes d'autoproduction du discours dont il vient d'être fait mention. On en citera deux exemples. Le premier est à chercher chez de Lancre qui, pour nommer le sabbat parle d' "Aquellare de verros". "Le lieu où on le trouve ordinairement s'appelle Lanne de bouc, \& en basque Aquellare de verros, prado del cabron, \& là les Sorciers le vont adorer trois nuicts durant, celle du Lundy, du Mercredy \& du Vendredy.» (1612:390) S'il est vrai que ce mot basque Akelarre désigne le " prado del cabrón ", le complément de nom «de verros » ne laisse pas d'interroger, d'autant qu'il n'est pas dans la Relación et pas non plus chez Pedro de Valencia. Dans le ${ }^{\circ} 15$ du Bulletin du Musée basque, Henri Gavel signale le caractère curieux de cette appellation qu'il rapproche d'une forme ancienne de berrocal et qui ferait donc référence à des rochers. La traduction serait «lande rocheuse du bouc ». Il réfute toute interprétation qui serait celle de « cresson » « qui ne semble pas fournir un sens satisfaisant » $(1938: 220)$. En effet, une piste, plus simple, semble plus prometteuse encore : selon la tradition, les réunions se tenaient dans un pré, proche de la grotte de Zugarramurdi, qui s'appelait Berroskoberro. La connaissance très rudimentaire $d u$ basque dont fait preuve de Lancre est vraisemblablement à l'origine de ce complément de nom. Cette appellation n'est pas parvenue à s'intégrer dans le schéma du stéréotype, mais on peut y voir comment le discours se nourrit d'apports divers dont l'origine s'efface complètement. D'une certaine façon, le discours démonologique fonctionne comme une machine à fabriquer du lieu commun : ce qui y est validé fait partie d'un répertoire où chaque auteur peut aller puiser.

L'autre exemple est chez Pedro de Valencia. Alors qu'il décrit, dans la Suma, le déroulement du sabbat, il établit des liens avec des cérémonies de l'Antiquité. Il fait référence aux Ménades, mais aussi aux mystères d'Éleusis, emploie notamment les termes telesa $a^{5}$ (en grec télétè, «initiation») ou epopteia (terme qui désigne le plus haut 
grade d'initiation lors des mystères d'Éleusis) ${ }^{6}$. La culture et l'érudition servent ici son propos de critique du discours.

Dicen que los de un aquelarre suelen ir á ver á los de otro quando están juntos lo qual es de notar por la costumbre antigua, que desde un convite salian calientes á dar grito y hacer chacota á los de otro por acrecentarles la fiesta, y estas idas se llamaban comessationes. » (1611a : fol. 10r)

L'analogie établie dans le corps du texte et non pas en note, il convient de le souligner, montre toute l'ampleur de ce que l'on peut qualifier, sans doute hâtivement, d'intertextualité. En montrant la similitude des motifs du sabbat et de la comissatio qui désigne, selon le dictionnaire Gaffiot, un «festin avec musique et danse suivi d'une promenade en cortège pour reconduire un des invités et recommencer la fête; partie de plaisir, orgie ", Pedro de Valencia montre combien le discours démonologique est apte à faire sienne une matière qui lui est étrangère, en d'autres termes à l'assimiler. Se souvient-on que l'accusation d'anthropophagie à l'égard des enfants était portée de façon récurrente contre les premiers chrétiens? Se souvient-on que le motif de la torche faite d'un bras d'enfant décrite dans la Relación est en fait une résurgence de ce motif magique connu comme main de gloire? Tous ces éléments ont été progressivement intégrés au discours pour, au détour d'une affaire, accéder au rang de témoignage, c'est-à-dire de preuve, donc de vérité. C'est que, dans le fond, le discours démonologique est de l'essence du mythe dont le caractère majeur est d'être répétitif " soit qu'il se répète à l'identique, soit que, remanié, il engendre un mythe de la même famille, mais d'une branche différente " (Kappler, $1980: 182$ ). Ce jugement porté sur l'image du monstre à la fin du Moyen Âge trouve dans la démonologie un nouveau champ d'application.

$\mathrm{Au}$ vrai, la seule marque qui condamne les sorcières et les sorciers n'est pas tant celle que laisserait le diable sur leur corps mais celle que leur inflige le juge en les accusant. On retiendra surtout de cette comparaison le fait que le discours démonologique n'est cela, un discours, mais qui, par son aspect totalitaire finit par s'imposer à la réalité elle-même. C'est ce qu'exprimait Feijoo, en 1728, dans le Teatro crítico (II, 5) : «En materia de hechicerías, como en la que más, circulan y se propagan las fábulas del Vulgo a los Escritores, y de los Escritores al Vulgo. » (Cité par Caro Baroja, 1967 : 313.) Dès lors, la seule posture possible est celle qu'avait adoptée le prêtre inquisiteur Alonso de Salazar y Frías qui, bien que juge au procès de Logroño, prônait de suspendre le jugement et surtout se taire, pour éviter de diffuser des récits dont la répétition sera vue comme un gage de vérité ; il sera, pour cela, et pour avoir convaincu la Suprema (le conseil suprême de l'Inquisition), qui reconnaît officiellement, le 31 août 1614, la fausseté des descriptions de sabbat, qualifié d'avocat des sorcières. Notre société, à l'heure des fake news et des réseaux sociaux, pourrait bien méditer cet exemple. 


\section{BIBLIOGRAPHIE}

BENREKASSA Georges (2007), «La preuve et l'aveu : ce que parler peut dire », Dix-huitième siècle, 39, 109-127, <https://doi.org/10.3917/dhs.039.0109>.

CARo BARoja Julio (1966), Las brujas y su mundo, Madrid : Alianza Editorial.

CARO BAROJA Julio (1967), Vidas mágicas e Inquisición, Madrid : Taurus.

CARo BARoja Julio (1970), Inquisición, brujería y criptojudaísmo, Barcelone : Ariel.

DUCROT Oswald (1983), « Opérateurs argumentatifs et visée argumentative », Cahiers de linguistique

française, 5, 7-36.

GAVel Henri (1938), « Notes sur le "Tableau sur l'inconstance des mauvais anges et des démons" de Pierre de Lancre », Bulletin du Musée basque, 15, <https://bmb.bilketa.eus/Bulletin-du-Museebasque-no15-1938?orria=89> (30 août 2019).

Gómiz LEón Juan José $(2013)$, « Moratín y las reediciones $(1811,1812)$ del Auto de Fe de Logroño », Dieciocho XVIII, 36(2), 329-338, <https://dieciocho-xviii.com/36.2/9.Gomiz\%20Leon.36.2.pdf> (septembre 2019 et 2021).

HENNINGSEN Gustav (1983), El abogado de las brujas. Brujería vasca e Inquisición española, Madrid : Alianza Editorial.

KAPPLER Claude (1980), Monstres, démons et merveilles à la fin du Moyen Âge, Paris : Payot.

KeRBRAT-ORECCHIONi Catherine (1980), L'énonciation, Paris : Armand Colin.

LANCRE Pierre de (1612), Tableau de l'inconstance des mauvais anges et démons où il est amplement traité des sorciers et de la Sorcellerie. Livre très-utile et nécessaire non seulement aux Iuges mais à tous ceux qui vivent sous les lois chrestiennes. Avec un discours contenant la procédure faicte par les Inquisiteurs d'Espagne \& de Navarre, à 53. Magiciens, Apostats, Iuifs \& Sorciers, en la ville de Logrogne en Castille, le 9. Nov. 1610. En laquelle on voit combien l'exercice de la justice en France est plus juridiquement traicté, \& avec de plus belles formes qu'en tous autres Empires, Royaumes, Republiques, \& Estats, Paris : chez Iean Berjon, rue S.Iean de Beauvais, au Cheval volant, \& en sa boutique au Palais à la galerie des prisonniers (source : gallica.bnf.fr / Bibliothèque nationale de France).

PÉREZ Joseph (2010), Historia de la brujería en España, Madrid : Espasa Calpe.

Relacion de las personas que salieron en el Auto de la Fee, que los señores Doctor Alonso Bezerra Holguin, del Abito de Alcantara: Licenciado Iuan de Valle Alvarado: Licenciado Alonso de Salazar Frias. Inquisidores Apostolicos, del Reyno de Navarra, y su distrito, celebraron en la Ciudad de Logroño, en siete, y en ocho dias del mes de Noviembre, de 1610 Años. Y de las cosas y delitos por que fueron castigados (in-8 ${ }^{\circ}$ ) (1611), Logroño : Iuan de Mongaston Impressor.

VALENCIA Pedro de (1611a), Suma de las relaciones de Logroño cerca de los brujos, hecha tambien por el mismo Pedro de Valencia, ms. ${ }^{0} 7579$ de la Bibliothèque nationale de Madrid, fol. 1-19v ${ }^{7}$.

VAlencia Pedro de (1611b), Discurso sobre los cuentos de las brujas, escrito por Pedro de Valencia.

Dirigido al Ill ${ }^{\text {mo }}$ Señor Don Bernardo de Sandoval y Rojas, Cardenal, Arçobispo de Toledo, Inquisidor General de España, ms. $\mathrm{n}^{\circ} 7579$ de la Bibliothèque nationale de Madrid, fol. 21-77v $v^{8}$. 


\section{NOTES}

1. Étymologiquement, le vocable «diable » vient d'un adjectif grec diabolos qui signifie «qui désunit, qui inspire la haine ou l'envie ». Substantivé, dans le vocabulaire sacré, il prend le sens que nous lui connaissons.

2. «Las onze personas que avian de ser relaxadas » (fol. 2).

3. À la référence ms. $\mathrm{n}^{\circ} 7579$ de la Biblioteca Nacional, correspondent la Suma de las relaciones de Logroño cerca de Brujos, hecha tambien por el mismo Pedro de Valencia (fol.1-19), citée ici, et le Discurso sobre los cuentos de las brujas, escrito por Pedro de Valencia. Dirigido al Ill ${ }^{\text {mo }}$ Señor Don Bernardo de Sandoval y Rojas, Cardenal, Arçobispo de Toledo, Inquisidor General de España, ms. n 7579 de la Bibliothèque nationale de Madrid, fol.21-77v. Ce sont des copies anonymes du XIX siècle, fidèlement reproduites d'après le manuscrit original aujourd'hui incomplet. Sur le sujet de l'établissement du texte, on consultera Gómiz León (2103) qui fait état d'éditions anciennes des deux textes (voir bibliographie).

4. Parti chasser, alors qu'il se trouvait à Bruges, Del Río vit sur un arbre des corbeaux et des pies sur lesquelles il tira, en atteignant une ; s'approchant de l'arbre il ne trouva qu'une clef qui se révéla être celle d'une maison dans laquelle le prêtre et un sien ami trouvèrent une femme blessée d'un coup d'arquebuse.

5. «Perfeccion de los misterios que llamaban telesas los griegos en los suyos. (Valencia, 1611 : fol. 7v)

6. «Tambien parece tiene semejanza con la Epopteia que se decia ansi al entrar los antiguos iniciados en los misterios ó estar viendo como se iniciaban otros nuevos. » (Valencia, 1611 : fol. 10r)

7. La bibliographie de Gómiz León (2013) relève une édition: Serrano y Sanz Manuel de (1906), "Segundo discurso de Pedro de Valencia acerca de los brujos y de sus maleficios. Suma de las relaciones de Logroño cerca de brujos, hecha también por el mismo Pedro de Valencia ", Revista de Archivos, Bibliotecas y Museos, XV(11-12), 445-454.

8. La bibliographie de Gómiz León (2013) fait aussi état d'une édition : Serrano y Sanz Manuel de (1900), « Discurso de Pedro de Valencia acerca de los quentos de las brujas y cosas tocantes a la magia ", Revista de Extremadura, 2, 289-303 et 337-347. On signalera aussi : Valencia Pedro de (1997), «Discurso acerca de los cuentos de las brujas», Obras completas VII, Estudios introductorios, notas y edición crítica por M. A. Marcos Casquero y H. B. Riesco Álvarez, León : Universidad de León.

\section{RÉSUMÉS}

La confrontation des commentaires de Pierre de Lancre et Pedro de Valencia sur le récit du procès de Logroño de novembre 1610 fait apparaître deux positions opposées quant à ce que l'on a appelé l'hérésie des sorcières. Le Français voit dans la récurrence des motifs la confirmation de l'existence de la sorcellerie alors que l'Espagnol choisit de ne considérer que l'aspect discursif pour en montrer les contradictions et l'irrationalité. Mais au-delà de la querelle juridique, c'est le statut du discours démonologique qui est en jeu: discours sur le monde ou pure construction? Au début du XVII ${ }^{\mathrm{e}}$ siècle, c'est en ces termes que se pose la question.

The confrontation of the comments of Pierre de Lancre and Pedro de Valencia on the account of the trial of Logroño (November 1610) reveals two opposing stances concerning what has been called the heresy of the witches. The French see in the recurrence of patterns the confirmation of 
the existence of witchcraft while the Spanish chooses to consider only the discursive aspect to show its contradictions and irrationality. Butbeyond the legal quarrel, the status of demonological discourse is at stake: discourse about the world or mere construction? In the early 17 th century, it was in these terms that the question arose.

INDEX

Keywords : speech analysis, witchcraft, Sabbath, Logroño, Pierre de Lancre, Pedro de Valencia Mots-clés : analyse de discours, sorcellerie, sabbat, Logroño, Pierre de Lancre, Pedro de Valencia

\section{AUTEUR}

RENAUD CAZALBOU

CEIIBA, EA 7412, UT2J

renaud.cazalbou@univ-tlse2.fr 